\title{
Successful management of major hemorrhage in 6 cases of abnormal invading placentation during 2016 in a tertiary hospital in Crete, Greece
}

Spartinou A., Christofaki M., Stefanakis G., Nyktari V., Tsagaraki G., Papaioannou A. General University Hospital of Heraklion, Dept of Anaesthesiology, Heraklion, Greece

\section{Introduction}

Major obstetric hemorrhage remains the leading cause of maternal morbidity and mortality worldwide. Among patients who receive massive transfusion, abnormal placentation is the most common etiology. There are three main entities (accreta, increta, percreta). Anaesthesiological management of six patients with abnormal placentation is presented.

\section{Cases Report}

Two cases of placentae accreta, two placentae increta and two placentae percreta in total of 882 pregnancies are reported. Demographic data are presented in table 1.

\begin{tabular}{|c|}
\hline Age \\
\hline ASA \\
\hline Gestation Age \\
\hline Gravida \\
\hline
\end{tabular}

$36.8(35-39)$ years

$33(30-36)$ weeks

$3(2-4)$

Table 1. Demographic data

Diagnosis of abnormal placentation was made prenatally with U/S and MRI. A multidisciplinary approach was decided for the successful management. Beyond the two senior anaesthetists and the two senior obstetricians, neonatalists, urologists, intensivists, and the blood bank personnel were included. Ureteral stents were placed preoperatively in increta/percreta placentae. General anesthesia with invasive monitoring was used (arterial catheter and two 16G peripheral IV catheters were placed in all cases, a central venous catheter was inserted only in cases where vasopressor therapy was required). Two grams of tranexamic acid were given post induction. Blood bank was informed for potential massive transfusion and 4 units of $\mathrm{RBCs}$ were brought to the operation theatre. Use of equipment for rapid administration of warmed fluids was readily available.

Hysterectomy with placenta in situ was preplanned in percreta cases, whereas in increta cases hysterectomy was performed due to severe haemorrhage. The uterus was preserved in accreta cases. In 2 cases urologists dealt with bladder invasion/ureter suturing. Massive transfusion protocol was activated in 5 cases (see Table 2). Serial lactate measurements guided fluid and vasopressor administration.
At the end of the operation, coagulation tests and PLT count were normal. Four patients were admitted to ICU for 24-48h with a further uncomplicated hospital stay.

\begin{tabular}{|c|c|c|c|c|c|c|c|}
$\begin{array}{c}\text { Placenta } \\
\text { Type }\end{array}$ & $\begin{array}{c}\text { Blood } \\
\text { loss } \\
\text { (L) }\end{array}$ & $\begin{array}{c}\text { Crystalloids } \\
\text { (L) }\end{array}$ & $\begin{array}{c}\text { RBC } \\
\text { (Units) }\end{array}$ & $\begin{array}{c}\text { FFP } \\
\text { (Units) }\end{array}$ & $\begin{array}{c}\text { PLT } \\
\text { (pool) }\end{array}$ & $\begin{array}{c}\text { Fibrinogen } \\
\text { (g) }\end{array}$ & $\begin{array}{c}\text { PCC } \\
\text { (IU) }\end{array}$ \\
\hline Percreta & 4.5 & 4 & 6 & 3 & 3 & 3 & - \\
\hline & 5.5 & 3 & 7 & 3 & 1 & 3 & 1000 \\
\hline Increta & 7 & 5,5 & 10 & 6 & 1 & 4 & 1000 \\
\hline & 5.5 & 3,0 & 7 & 5 & 1 & 4 & - \\
\hline Accreta & 1.2 & 1,5 & 3 & - & - & 3 & - \\
\hline & 4.5 & 3.5 & 6 & 5 & - & 2 & - \\
\hline
\end{tabular}

Table 2

\section{Discussion}

Invading placentae can lead to catastrophic haemorrhage. The incidence of abnormal placentation has increased to $1: 533$ gestations ${ }^{1}$. Our hospital receives all cases of invading placentae from South Greece. This might explain the increased percentage of abnormal placentation in our institution (6:882 pregnancies).

Risk factors include advanced maternal age, multiple gestations, placenta previa and previous uterine surgery, all of which occurred in the cases presented ${ }^{1}$.

Due to lack of point-of-care devices for accessing coagulopathy, massive transfusion is based on local protocol. Early administration of tranexamic acid (20-25 $\mathrm{mg} / \mathrm{kg}$ ) and fibrinogen (3-4gr) are the key points. Prompt use of PCCs had also an important role in major bleeding. A fibrinogen level $>200 \mathrm{mg} / \mathrm{dl}$ was targeted, as lower level is regarded as predictor of peripartum haemorrhage ${ }^{2}$.

Recent evidence has shown that outcome can be improved with the use of novel interventional radiology, when this is available 1 .

Prenatal diagnosis, multidisciplinary approach and prompt use of massive transfusion protocol allow safe and successful delivery, despite limited resources.

\section{$\underline{\text { References }}$}

1. Center of excellence for placenta accreta, Silver RM et al. Am J Obstet Gynecol.2015 May;212(5):5618

2. Transfusion and coagulation management in major obstetric haemorrhage. Butwick AJ et al. Curr Opin Anaesthesiol.2015 Jun;28(3):27584 